\title{
Validation of the Updated Renal Graded Prognostic Assessment (GPA) for patients with renal cancer brain metastases treated with Gamma Knife radiosurgery
}

\author{
Niels van Ruitenbeek ( $\sim$ n.vanruitenbeek@etz.nl) \\ Elisabeth-TweeSteden Ziekenhuis https://orcid.org/0000-0002-5122-6021 \\ Vincent Ho \\ Comprehensive Cancer Centre Netherlands: Integraal Kankercentrum Nederland \\ Hans Westgeest \\ Amphia Hospital: Amphia Ziekenhuis \\ Laurens Beerepoot \\ Elisabeth-TweeSteden Ziekenhuis \\ Patrick Hanssens \\ Elisabeth-TweeSteden Ziekenhuis
}

\section{Research Article}

Keywords: Gamma Knife radiosurgery, Brain metastasis, Renal cell carcinoma, Prognosis

Posted Date: April 16th, 2021

DOI: https://doi.org/10.21203/rs.3.rs-395538/v1

License: (c) (i) This work is licensed under a Creative Commons Attribution 4.0 International License. Read Full License 


\section{Abstract}

Introduction: Prognosis of patients with brain metastasis (BM) from renal cell carcinoma (RCC) is relevant for treatment decisions and can be estimated with the Renal Graded Prognostic Assessment (GPA). The aim of this study is to validate the updated version of this instrument in a cohort treated with Gamma Knife radiosurgery (GKRS) without prior local intracerebral therapy.

Methods: Between 2007 and 2018, 100 RCC patients with BM were treated with GKRS. They were categorized according to the updated Renal GPA. Overall survival (OS), intracranial disease progression and intracranial local failure were estimated using the Kaplan-Meier method and risk factors were identified with Cox proportional hazard regressions.

Results: Median OS was 10.4 months. Median OS for GPA categories 0.0-1.0 (10\%), 1.5-2.0 (13\%), 2.5-3.0 (37\%) and 3.5-4.0 (31\%) was 2.9, 5.5, 8.1 and 20.4 months, respectively. Karnofsky performance status $<90$, serum hemoglobin $\leq 12.5 \mathrm{~g} / \mathrm{dL}$, age $>65 \mathrm{years}$ and time from primary diagnosis to brain metastasis $<1$ year were significantly related with shorter survival, while presence of extracranial disease, the volume and total number of BM had no impact on OS. A total count of $>4$ BM was the only predictive factor for intracranial disease progression, while none of the investigated factors predicted intracranial local failure.

Conclusions: This study confirms the updated Renal GPA in an independent cohort as a valuable instrument to estimate survival in patients with BM from RCC treated with GKRS.

\section{Introduction}

Metastatic renal cell carcinoma (RCC) is a relatively rare disease, with about 900 patients per year in the Netherlands [1]. Approximately $8 \%-28 \%$ of patients with metastatic RCC will develop brain metastases (BM) [2-4]. This incidence has been rising due to earlier detection with more sensitive imaging techniques combined with increased survival as a result of more effective systemic therapies. Novel first-line treatments include vascular endothelial growth factor (VEGF) targeted tyrosine kinase inhibitor (TKI) monotherapy [5], dual immune checkpoint inhibitor (ICI) therapy [6] and ICI/TKI combination therapy [7], which were approved in the Netherlands in 2007, 2019 and 2020, respectively. Beyond first-line, ICI monotherapy became available in 2016. Decisions about systemic treatment type are largely based on International Metastatic RCC Database Consortium (IMDC) criteria [8] and histological subtype. The development of new systemic therapy has led to improved survival of RCC patients with $\mathrm{BM}$ reaching 14.4 months in selected populations $[9,10]$. However, patients with BM were excluded from the pivotal trials of the novel systemic therapies.

Intracranial treatment options for RCC BM include neurosurgical resection with or without postoperative tumor bed irradiation, whole brain radiotherapy (WBRT) and stereotactic radiosurgery (SRS), including Gamma Knife radiosurgery (GKRS). RCC BM are relatively resistant to WBRT with radiation delivered in conventional fractionated doses [11]. SRS delivers high radiation dose to visible BM, sparing the surrounding healthy brain tissue [12]. In contrast to WBRT, SRS generally achieves local tumor control with limited neurotoxicity [13], at the cost of an increased risk of new visible BM when given alone [14]. To address this risk, radiosurgery is best combined with subsequent systemic therapy for prevention of new BM and with surveillance screening for early detection and prompt treatment of local recurrences or new BM. In cohorts of patients with RCC BM treated with GKRS, local tumor control after GKRS was achieved in $92 \%$ of patients, while survival rates varied significantly [15, 16].

For clinicians, accurate estimation of survival of patients with BM treated with SRS remains a challenge [17]. Prognostic risk scores can be useful in counselling patients about treatment. One frequently used prognostic risk score is the diagnosis-specific Graded Prognostic Assessment (GPA), with the Renal GPA specifically developed for RCC. The first version of this instrument was based on a 1985-2007 cohort, which was in the era before VEGF targeted TKI treatment [18], and was later validated in a 1998-2011 GKRS cohort [19]. Recently, the Renal GPA has been updated based on a more recent 2006-2015 cohort [20]. The updated Renal GPA stratifies patients in prognostic groups, based on Karnofsky performance status, number of BM, the presence of extracranial metastases and serum hemoglobin level.

However, the treatment approaches for BM used in the cohort of the updated Renal GPA were heterogeneous. Therefore, it is unknown whether this prediction score is valid for patients treated with GKRS. In this study, we independently validate the updated Renal GPA in patients with RCC and BM treated with GKRS. Also, we investigate additional pre-treatment factors that may independently predict for overall survival (OS), intracranial disease progression (IDP) and intracranial local failure (ILF). Furthermore, an attempt will be made to correlate type of systemic treatment with OS and intracranial disease development.

\section{Methods}

\section{Data collection}

After approval of the institutional review board, a cohort of all RCC patients with newly discovered BM who were treated with GKRS in the Gamma Knife Center Tilburg, Elisabeth-TweeSteden (ETZ) Hospital, Tilburg, The Netherlands, in the years 2007-2018 was retrospectively studied. 
Patients who underwent prior neurosurgery, radiosurgery or radiotherapy, patients with Karnofsky performance status $<70$ and patients with more than one active primary malignancy were excluded. Data were collected and saved in a coded database.

Patient, disease and treatment characteristics were retrieved from patient records and GammaPlan software ${ }^{\circledR}$ treatment records. Activity of extracranial disease was divided into three categories: active, stable or absent, based on whole-body CT-scans within two months from BM diagnosis. Active disease was defined as progressive or new burden of disease outside the brain. Patients with BM as first manifestation concurrent with extracranial disease were also classified as having active disease. Stable disease denoted previously treated extracranial burden of disease responsive to treatment, marked by partial response or stable disease. Absent extracranial disease indicated patients without a history of extracranial metastases or previously treated extracranial disease, including (partial) nephrectomy, with complete radiographic response.

Synchronous BM were defined as those occurring within one month of RCC diagnosis. Values of serum hemoglobin were obtained from the date nearest to the date of BM diagnosis, with a maximum difference of six weeks.

The updated Renal GPA was calculated for each patient according to the original study [20]. In case of missing extracranial disease status, no Renal GPA was calculated. These patients were not excluded from further analysis, in order to give an accurate portrayal of our patient cohort.

\section{Gamma Knife radiosurgery}

GKRS was conducted as an outpatient procedure. A Leksell® stereotactic G-frame was applied to the skull under local anesthesia. A high resolution planning-MRI scan of the brain without and with triple dose of gadolinium was performed. Radiosurgical planning was made by a neurosurgeon, radiation oncologist and physicist using Leksell GammaPlan software® (Elekta Instrument, Stockholm, Sweden). GKRS was performed using the Leksell Gamma Unit Model 4 C® until December 2008, and Perfexion®/ ICON® (Elekta AB, Stockholm, Sweden) thereafter. Depending on volume and location, a dose of 18-25 Gy was prescribed to the isodoseline covering $99-100 \%$ of the tumor volume. First clinical and radiological follow-up was routinely performed 6 weeks to 3 months after GKRS, the follow-ups thereafter were requested at 6 weeks-3 months intervals as long as clinically relevant. In case of new visible BM at surveillance, retreatment was performed.

\section{Statistical analysis}

OS was defined as the time between first GKRS and death, or the time between first GKRS and date of censoring in case patients were still alive. IDP was defined as development of new BM and/or leptomeningeal disease at sites other than previous treatment. ILF was defined as radiological local progression of the tumor after GKRS. ILF was determined through radiological features. Any contrast enhancing increase in volume of a treated BM compared to its volume on a previous MRI scan was considered as ILF once pseudoprogression or adverse radiation effects were ruled out based on the characteristics on T2 weighted images, on pre- and post-contrast T1 weighted images and on perfusion MRI. Patients without any failure were censored at the date of last imaging showing intracranial control. Maximum follow-up for OS, IDP and ILP was 60 months after GKRS.

Univariable and multivariable analyses were performed using Cox proportional hazards regression in order to identify variables in our data set that were significantly associated with OS, IDP and ILF.

The variables demonstrating association $(p \leq 0.20)$ on univariable analysis were selected for multivariable analysis. Highly correlated variables $(r$ $>0.7$ ) were not selected. A p-value of $<0.05$ was considered statistically significant.

Differences in OS and IDP between different types and timing of systemic therapy were analyzed using multivariable Cox proportional hazards regression. Variables demonstrating association $(p \leq 0.20)$ with OS and IDP in univariable analyses were selected for multivariable analysis together with the different types and timing of systemic therapies. Three timings of systemic therapy were defined: 'before', 'after' and 'before and after'. Patients who received a type of systemic therapy before (i.e., 'before' and 'before and after') or after (i.e. 'after' and 'before and after') GKRS were compared with those who did not receive this therapy. Patients who received systemic therapy within one month after GKRS were placed in the 'before and after' group. Data about systemic therapy were not available in one patient, who was excluded from this analysis.

Survival curves of the Renal GPA groups were estimated by the Kaplan-Meier method and compared by means of the log-rank test. Analyses were performed using SPSS software (version 24.0) and graphs were created using GraphPad Prism software (version 5.03).

\section{Results}

\section{Patient characteristics}

A total of 100 patients who were treated with GKRS for newly discovered RCC BM were identified. Patient characteristics and median survival by subgroups are presented in Table 1. Seventy-eight patients (78\%) were male. At the time of the first GKRS treatment, median age was 65 (interquartile range [IQR] 56-69). Sixty-nine patients (69\%) had performance status of 90 or higher, and 67 patients (74\%) had active extracranial disease. The median time between RCC diagnosis and first BM was 15.0 (IQR 3.0-47.6) months. Patients underwent GKRS to a median of two (IQR 1-4) metastases. Forty-five patients (45\%) received systemic treatment before and after GKRS. Serum hemoglobin was obtained in 85 
patients (85\%) and was not available in the remainder. Data to calculate Renal GPA scores were available in 91 patients (91\%). The mean and median updated Renal GPA scores were 2.7 and 3.0, respectively (IQR 2.0-3.5). 
Patients characteristics and median survival

\begin{tabular}{|c|c|c|c|}
\hline Variable & Category & Number of patients (\%) & $\begin{array}{l}\text { Median survival in months } \\
\text { (IQR) }\end{array}$ \\
\hline Overall & & 100 & $10.4(4.1,26.1)$ \\
\hline \multirow[t]{2}{*}{ Gender } & Male & $78(78 \%)$ & $11.0(4.8,26.1)$ \\
\hline & Female & $22(22 \%)$ & $4.9(3.0,34.2)$ \\
\hline \multirow[t]{4}{*}{ Age } & Median [range] & 65 [37-85] & \\
\hline & $37-55$ & $24(24 \%)$ & $7.2(4.6,24.7)$ \\
\hline & $55-65$ & $33(33 \%)$ & $13.8(6.5,46.0)$ \\
\hline & $66-85$ & $43(43 \%)$ & $7.4(3.0,15.2)$ \\
\hline \multirow[t]{6}{*}{ Tumor histology } & Clear cell & $80(80 \%)$ & $11.0(4.9,29.5)$ \\
\hline & $\begin{array}{l}\text { Clear cell, sarcomatoid } \\
\text { dedifferentiation }\end{array}$ & $3(3 \%)$ & 0.6 \\
\hline & Chromophobe & $2(2 \%)$ & 0.2 \\
\hline & Papillary & $1(1 \%)$ & 4.5 \\
\hline & Renal cell carcinoma, NOS & $11(11 \%)$ & $13.8(2.6,52.3)$ \\
\hline & Radiodiagnostic diagnosis & $3(3 \%)$ & 3.8 \\
\hline \multirow[t]{5}{*}{ Fuhrmann grade } & 1 & $5(5 \%)$ & $13.4(0.8,21.3)$ \\
\hline & 2 & $26(26 \%)$ & $8.0(2.7,26.1)$ \\
\hline & 3 & $22(20.8 \%)$ & $10.4(6.4,26.2)$ \\
\hline & 4 & $10(10.4 \%)$ & $7.4(5.9,24.4)$ \\
\hline & Not available & $37(37 \%)$ & $8.6(4.1,20.9)$ \\
\hline \multirow[t]{3}{*}{ Kamofsky performance status } & Median [range] & $90[70-100]$ & \\
\hline & $70-80$ & $31(31 \%)$ & $5.5(2.2,10.6)$ \\
\hline & $90-100$ & $69(69 \%)$ & $13.4(6.4,33.3)$ \\
\hline \multirow[t]{4}{*}{ BM number } & Median [range] & $2[1-13]$ & \\
\hline & 1 & $39(39 \%)$ & $10.4(4.5,29.5)$ \\
\hline & $2-4$ & $40(40 \%)$ & $10.6(3.7,17.5)$ \\
\hline & $>4$ & $21(21 \%)$ & $7.8(4.6,34.2)$ \\
\hline \multirow[t]{3}{*}{ BM total volume $\left(\mathrm{cm}^{3}\right)$} & Median [range] & $4.5[0.14-36.9]$ & \\
\hline & $\leq 10$ & $81(81 \%)$ & $10.4(4.1,26.1)$ \\
\hline & $>10$ & $19(19 \%)$ & $11.3(5.9,29.5)$ \\
\hline \multirow[t]{4}{*}{ Extracranial disease status } & Absent & $8(8 \%)$ & $20.4(3.4,75.2)$ \\
\hline & Stable & $16(16 \%)$ & $17.2(4.8,52.3)$ \\
\hline & Active & $67(67 \%)$ & $7.8(3.8,19.1)$ \\
\hline & Not available & $9(9 \%)$ & $13.0(10.2,26.2)$ \\
\hline \multirow[t]{2}{*}{ Primary tumor resection } & Yes & $79(79 \%)$ & $10.6(4.7,26.2)$ \\
\hline & No & $21(21 \%)$ & $7.2(3.7,19.1)$ \\
\hline \multicolumn{4}{|c|}{$I Q R$ interquartile range, NOS not otherwise specified, $B M$ brain metastasis, } \\
\hline \multicolumn{4}{|c|}{ TPDBM time from primary diagnosis to brain metastasis, GKRS Gamma Knife radiosurgery, } \\
\hline GPA graded prognostic assessr & & & \\
\hline
\end{tabular}




\begin{tabular}{|c|c|c|c|}
\hline Variable & Category & Number of patients (\%) & $\begin{array}{l}\text { Median survival in months } \\
\text { (IQR) }\end{array}$ \\
\hline \multirow[t]{5}{*}{ TPDBM } & Median [range] & $15.0[0-247.2]$ & \\
\hline & Synchronous brain metastasis & $21(21 \%)$ & $7.4(4.6,20.9)$ \\
\hline & 1 month-1 year & $26(26 \%)$ & $4.6(2.2,11.0)$ \\
\hline & $1-4$ years & $27(27 \%)$ & $7.8(4.7,75.2)$ \\
\hline & $>4$ years & $26(26 \%)$ & $14.7(10.6,46.0)$ \\
\hline \multirow[t]{5}{*}{ Hemoglobin (g/dL; mmol/l) } & Median [range] & $\begin{array}{l}13.0[4.7,18.5] ; 8.1[2.9 \\
11.5]\end{array}$ & \\
\hline & $<11.2 ;<6.9$ & $16(16 \%)$ & $4.6(2.6,7.4)$ \\
\hline & $11.2-12.5 ; 6.9-7.8$ & $16(16 \%)$ & $4.5(2.2,10.7)$ \\
\hline & $>12.5 ;>7.8$ & $53(53 \%)$ & $14.7(7.8,34.2)$ \\
\hline & Not available & $15(15 \%)$ & $5.6(0.8,19.1)$ \\
\hline \multirow{3}{*}{$\begin{array}{l}\text { Systemic treatment before and after } \\
\text { GKRS }\end{array}$} & Yes & $45(45 \%)$ & $10.4(5.5,33.3)$ \\
\hline & No & $54(54 \%)$ & $10.6(3.4,24.4)$ \\
\hline & Not available & $1(1 \%)$ & 4.5 \\
\hline \multirow[t]{5}{*}{ Number of GKRS courses } & Mean [range] & $1.7[1-11]$ & \\
\hline & 1 & $65(65 \%)$ & $6.5(3.1,14.7)$ \\
\hline & 2 & $19(19 \%)$ & $13.0(7.8,34.2)$ \\
\hline & 3 & $7(7 \%)$ & $46.0(17.5,52.3)$ \\
\hline & $>3$ & $9(9 \%)$ & $33.3(20.6,81.9)$ \\
\hline \multirow[t]{6}{*}{ Updated Renal GPA } & Median; mean & $3.0 ; 2.7$ & \\
\hline & $0.0-1.0$ & $10(10 \%)$ & $2.9(2.2,7.1)$ \\
\hline & $1.5-2.0$ & $13(13 \%)$ & $5.5(4.6,10.6)$ \\
\hline & $2.5-3.0$ & $37(37 \%)$ & $8.1(4.1,20.6)$ \\
\hline & $3.5-4.0$ & $31(31 \%)$ & $20.4(8.4,38.6)$ \\
\hline & Not available & $9(9 \%)$ & $13.0(10.2,26.2)$ \\
\hline \multicolumn{4}{|c|}{$I Q R$ interquartile range, NOS not otherwise specified, $B M$ brain metastasis, } \\
\hline \multicolumn{4}{|c|}{ TPDBM time from primary diagnosis to brain metastasis, GKRS Gamma Knife radiosurgery, } \\
\hline \multicolumn{4}{|l|}{ GPA graded prognostic assessment } \\
\hline
\end{tabular}

\section{Overall survival}

With a median follow-up of 48.9 (IQR 32.4-60.0) months after GKRS, mOS of this cohort was 10.4 (IQR 4.1-26.1) months. When stratified according to the updated Renal GPA, mOS in the category with scores of 0.0-1.0 was 2.9 (IQR 2.2-7.1) months. Patients with scores of 1.5-2.0 had mOS of 5.5 (IQR 4.6-10.6) months, while mOS was 8.1 (IQR 4.1-20.6) months in the 2.5-3.0 category, and 20.4 (IQR 8.4-38.6) months in the 3.5-4.0 class. Kaplan-Meier curves showing OS for each Renal GPA group are displayed in Fig. 1A. Patients with Renal GPA scores of 0.0-1.0 had significantly shorter survival than patients with scores of $>1.0$ (mOS 2.9 vs 10.6 months; $p<0.01$ ) (Fig. 1B). Patients with scores of 3.5-4.0 had significantly longer survival than patients with Renal GPA $<3.5$ (mOS 20.4 vs 6.7 months; $p=0.03$ ) (Fig. 1C).

Univariable analysis identified the following factors to be associated with worse survival outcome: age $>65$ years, performance status $70-80$, presence of extracranial disease, serum hemoglobin $<12.5 \mathrm{~g} / \mathrm{dL}[<7.8 \mathrm{mmol} / \mathrm{l}$, and time from primary diagnosis to brain metastasis $<1$ year. On multivariable analysis, factors associated with worse survival outcome included age $>65$ years (HR 2.2; $p<0.01$ ), performance status $70-80$ (HR 2.6; $p<0.01)$, serum hemoglobin $<12.5 \mathrm{~g} / \mathrm{dL}[<7.8 \mathrm{mmol} / \mathrm{l}](\mathrm{HR} 2.3 ; \mathrm{p}<0.01)$, and time from primary diagnosis to brain metastasis $<1$ year $(\mathrm{HR}$ 
$2.0 ; \mathrm{p}<0.01)$. Patients with present extracranial disease had worse OS than those with absent extracranial disease, although not significant $(\mathrm{HR}$ 2.4; $p=0.07$ ) (Table 2). 
Table 2

Univariable and multivariable Cox proportional hazard analysis for overall survival

Overall survival

Intracranial distant progression

Intracranial local failure

\begin{tabular}{|c|c|c|c|c|c|c|c|c|c|c|c|c|c|c|c|c|c|}
\hline \multirow[b]{2}{*}{ Factor } & \multirow[b]{2}{*}{ Category } & \multirow[b]{2}{*}{ C } & \multirow[b]{2}{*}{$E$} & \multicolumn{2}{|c|}{ Univariable } & \multicolumn{2}{|c|}{ Multivariable } & \multirow[b]{2}{*}{ C } & \multirow[b]{2}{*}{$\mathbf{E}$} & \multicolumn{2}{|c|}{ Univariable } & \multicolumn{2}{|c|}{ Multivariable } & \multirow[b]{2}{*}{ C } & \multirow[b]{2}{*}{$E$} & \multicolumn{2}{|c|}{ Univariable } \\
\hline & & & & HR & $P$ & HR & $\mathbf{P}$ & & & HR & $\mathbf{P}$ & $\mathrm{HR}$ & $\mathbf{P}$ & & & $\mathrm{HR}$ & $P$ \\
\hline & & a & $\mathbf{v}$ & $\begin{array}{l}(95 \% \\
\mathrm{Cl})\end{array}$ & & $\begin{array}{l}(95 \% \\
\mathrm{Cl})\end{array}$ & & a & $\mathbf{v}$ & $\begin{array}{l}(95 \% \\
\text { Cl) }\end{array}$ & & $\begin{array}{l}(95 \% \\
\mathrm{Cl})\end{array}$ & & a & $\mathbf{v}$ & $\begin{array}{l}(95 \% \\
\mathrm{Cl})\end{array}$ & \\
\hline & & s & e & & & & & s & e & & & & & s & e & & \\
\hline & & e & $\mathrm{n}$ & & & & & e & $\mathrm{n}$ & & & & & e & $n$ & & \\
\hline & & $\mathbf{s}$ & $t$ & & & & & $\mathbf{s}$ & $t$ & & & & & $\mathbf{s}$ & $t$ & & \\
\hline & & & $\mathbf{s}$ & & & & & & $\mathbf{s}$ & & & & & & $\mathbf{s}$ & & \\
\hline \multirow[t]{3}{*}{ Age } & $\leq 65$ & 57 & 42 & $\begin{array}{l}1.0 \\
\text { (Ref) }\end{array}$ & - & $\begin{array}{l}1.0 \\
\text { (Ref) }\end{array}$ & - & 49 & 34 & $\begin{array}{l}1.0 \\
\text { (Ref) }\end{array}$ & - & $\begin{array}{l}1.0 \\
\text { (Ref) }\end{array}$ & - & 49 & 4 & $\begin{array}{l}1.0 \\
\text { (Ref) }\end{array}$ & - \\
\hline & $>65$ & 43 & 41 & 1.9 & $<$ & & $<01$ & 32 & 14 & 0.6 & 0.17 & 0.7 & 0.21 & 32 & 3 & 1.0 & 0.99 \\
\hline & & & & $\begin{array}{l}(1.2 \\
2.9)\end{array}$ & & $\begin{array}{l}(1.3 \\
3.5)\end{array}$ & & & & $\begin{array}{l}(0.3, \\
1.2)\end{array}$ & & $\begin{array}{l}\text { (0.4, } \\
1.3)\end{array}$ & & & & $\begin{array}{l}(0.3 \\
4.0)\end{array}$ & \\
\hline \multirow[t]{3}{*}{ Gender } & Male & 78 & 63 & $\begin{array}{l}1.0 \\
\text { (Ref) }\end{array}$ & - & - & - & 66 & 41 & $\begin{array}{l}1.0 \\
\text { (Ref) }\end{array}$ & - & - & - & 66 & 8 & $\begin{array}{l}1.0 \\
\text { (Ref) }\end{array}$ & - \\
\hline & Female & 22 & 20 & 1.3 & 0.36 & - & - & 15 & 7 & 0.6 & 0.22 & - & - & 15 & 2 & 1.1 & 0.91 \\
\hline & & & & $\begin{array}{l}(0.8 \\
2.1)\end{array}$ & & & & & & $1.3)^{\prime}$ & & & & & & $\begin{array}{l}(0.2 \\
5.2)\end{array}$ & \\
\hline \multirow{3}{*}{$\begin{array}{l}\text { Kamofsky } \\
\text { performance } \\
\text { status* }\end{array}$} & $70-80$ & 31 & 27 & 1.9 & 0.01 & 2.6 & $<$ & 20 & 9 & 0.9 & 0.69 & - & - & 20 & 1 & 0.5 & 0.54 \\
\hline & & & & $\begin{array}{l}(1.2 \\
3.0)\end{array}$ & & $\begin{array}{l}(1.6 \\
4.3)\end{array}$ & & & & $\begin{array}{l}(0.4 \\
1.8)\end{array}$ & & & & & & $\begin{array}{l}(0.1 \\
4.4)\end{array}$ & \\
\hline & $90-100$ & 69 & 36 & $\begin{array}{l}1.0 \\
\text { (Ref) }\end{array}$ & - & $\begin{array}{l}1.0 \\
\text { (Ref) }\end{array}$ & - & 61 & 39 & $\begin{array}{l}1.0 \\
\text { (Ref) }\end{array}$ & - & - & - & 61 & 9 & $\begin{array}{l}1.0 \\
\text { (Ref) }\end{array}$ & - \\
\hline \multirow[t]{5}{*}{ BM number* } & 1 & 39 & 31 & $\begin{array}{l}1.0 \\
\text { (Ref) }\end{array}$ & - & - & - & 34 & 16 & $\begin{array}{l}1.0 \\
\text { (Ref) }\end{array}$ & - & $\begin{array}{l}1.0 \\
\text { (Ref) }\end{array}$ & & 34 & 5 & $\begin{array}{l}1.0 \\
\text { (Ref) }\end{array}$ & - \\
\hline & $2-4$ & 40 & 36 & 1.3 & 0.24 & - & - & 31 & 19 & 1.5 & 0.26 & 1.5 & 0.27 & 31 & 3 & 0.7 & 0.62 \\
\hline & & & & $\begin{array}{l}(0.8 \\
2.2)\end{array}$ & & & & & & $\begin{array}{l}(0.8 \\
2.9)\end{array}$ & & $\begin{array}{l}(0.7 \\
2.8)\end{array}$ & & & & $\begin{array}{l}(0.2 \\
2.9)\end{array}$ & \\
\hline & $>4$ & 21 & 16 & 1.0 & 0.93 & - & - & 16 & 13 & 2.3 & 0.03 & 2.2 & 0.04 & 16 & 3 & 0.8 & 0.77 \\
\hline & & & & $\begin{array}{l}(0.6 \\
1.9)\end{array}$ & & & & & & $\begin{array}{l}(1.1 \\
4.8)\end{array}$ & & $\begin{array}{l}(1.1 \\
4.7)^{\prime}\end{array}$ & & & & $\begin{array}{l}(0.2 \\
4.1)\end{array}$ & \\
\hline \multirow{3}{*}{$\begin{array}{l}\text { BM total } \\
\text { volume } \\
\left(\mathrm{cm}^{3}\right)^{\star}\end{array}$} & $\leq 10$ & 81 & 19 & $\begin{array}{l}1.0 \\
\text { (Ref) }\end{array}$ & - & - & - & 65 & 40 & $\begin{array}{l}1.0 \\
\text { (Ref) }\end{array}$ & - & - & - & 65 & 7 & $\begin{array}{l}1.0 \\
\text { (Ref) }\end{array}$ & - \\
\hline & $>10$ & 19 & 16 & 1.0 & 0.97 & - & - & 16 & 8 & 0.7 & 0.28 & - & - & 16 & 3 & 1.7 & 0.45 \\
\hline & & & & $\begin{array}{l}\text { (0.6) } \\
1.7)^{2}\end{array}$ & & & & & & $\begin{array}{l}\text { (0.3) } \\
1.4)^{2}\end{array}$ & & & & & & $\begin{array}{l}(0.4 \\
6.6)\end{array}$ & \\
\hline $\begin{array}{l}\text { Extracranial } \\
\text { disease } \\
\text { status }\end{array}$ & Absent & 9 & 5 & $\begin{array}{l}1.0 \\
\text { (Ref) }\end{array}$ & - & $\begin{array}{l}1.0 \\
\text { (Ref) }\end{array}$ & - & 8 & 6 & $\begin{array}{l}1.0 \\
\text { (Ref) }\end{array}$ & - & - & - & 8 & 1 & $\begin{array}{l}1.0 \\
\text { (Ref) }\end{array}$ & - \\
\hline
\end{tabular}

$H R$ hazard ratio, $B M$ brain metastasis, TPDBM time from primary diagnosis to brain metastasis

Bold text indicates statistical significance.

* Cut-off values selected because of clinical relevance. 


\begin{tabular}{|c|c|c|c|c|c|c|c|c|c|c|c|c|c|c|c|c|c|}
\hline & \multicolumn{7}{|c|}{ Overall survival } & \multicolumn{6}{|c|}{ Intracranial distant progression } & \multicolumn{4}{|c|}{ Intracranial local failure } \\
\hline & \multirow[t]{2}{*}{ Present } & \multirow[t]{2}{*}{82} & \multirow[t]{2}{*}{70} & 2.6 & \multirow[t]{2}{*}{0.04} & \multirow{2}{*}{$\begin{array}{l}2.4 \\
(0.9 \\
6.3)\end{array}$} & \multirow[t]{2}{*}{0.07} & \multirow[t]{2}{*}{66} & \multirow[t]{2}{*}{37} & \multirow{2}{*}{$\begin{array}{l}1.0 \\
(0.4, \\
2.3)\end{array}$} & \multirow[t]{2}{*}{0.96} & \multirow[t]{2}{*}{-} & \multirow[t]{2}{*}{-} & \multirow[t]{2}{*}{66} & \multirow[t]{2}{*}{8} & 2.0 & \multirow[t]{2}{*}{0.53} \\
\hline & & & & $\begin{array}{l}(1.1 \\
6.6)\end{array}$ & & & & & & & & & & & & $\begin{array}{l}(0.2, \\
15.8)\end{array}$ & \\
\hline & \multirow{2}{*}{$\begin{array}{l}\text { Not } \\
\text { available }\end{array}$} & \multirow[t]{2}{*}{9} & \multirow[t]{2}{*}{8} & 2.5 & \multirow[t]{2}{*}{0.12} & 4.4 & \multirow[t]{2}{*}{0.02} & \multirow[t]{2}{*}{7} & \multirow[t]{2}{*}{5} & 1.1 & \multirow[t]{2}{*}{0.87} & \multirow[t]{2}{*}{-} & \multirow[t]{2}{*}{-} & \multirow[t]{2}{*}{7} & \multirow[t]{2}{*}{4} & 1.7 & \multirow[t]{2}{*}{0.70} \\
\hline & & & & $\begin{array}{l}(0.8, \\
7.6)\end{array}$ & & $\begin{array}{l}(1.3, \\
14.0)\end{array}$ & & & & $\begin{array}{l}(0.3 \\
3.7)\end{array}$ & & & & & & $\begin{array}{l}(0.1 \\
28.5)\end{array}$ & \\
\hline \multirow{6}{*}{$\begin{array}{l}\text { Hemoglobin } \\
(\mathrm{g} / \mathrm{dL} ; \\
\mathrm{mmol} / \mathrm{l})\end{array}$} & $\leq 12.5$ & 31 & 28 & 2.3 & $<01$ & 2.3 & $<01$ & 19 & 8 & 1.2 & 0.62 & - & - & 19 & 7 & 0.9 & 0.89 \\
\hline & $\leq 7.8$ & & & $\begin{array}{l}(1.4, \\
3.7)\end{array}$ & & $\begin{array}{l}(1.4, \\
3.9)\end{array}$ & & & & $\begin{array}{l}(0.6 \\
2.7)\end{array}$ & & & & & & $\begin{array}{l}(0.1 \\
7.0)^{\prime}\end{array}$ & \\
\hline & $>12.5$ & 54 & 42 & 1.0 & - & 1.0 & - & 51 & 31 & 1.0 & - & - & - & 51 & 1 & 1.0 & - \\
\hline & $>7.8$ & & & & & & & & & & & & & & & & \\
\hline & $\begin{array}{l}\text { Not } \\
\text { available }\end{array}$ & 15 & 13 & 1.7 & 0.08 & 3.6 & $<01$ & 11 & 9 & 1.8 & 0.11 & - & - & 11 & 2 & 1.5 & 0.63 \\
\hline & & & & $\begin{array}{l}(0.9 \\
3.3)\end{array}$ & & $7.2)^{\prime}$ & & & & $\begin{array}{l}(0.9 \\
3.9)\end{array}$ & & & & & & $\begin{array}{l}(0.3, \\
7.1)\end{array}$ & \\
\hline $\begin{array}{l}\text { TPDBM } \\
\text { (vears)* }\end{array}$ & $<1$ & 47 & 42 & 1.9 & <. & 2.0 & $<$ & 37 & 20 & 1.4 & 0.24 & - & - & 37 & 6 & 3.2 & 0.08 \\
\hline & & & & $\begin{array}{l}(1.2 \\
2.9)\end{array}$ & & $\begin{array}{l}(1.3, \\
3.2)\end{array}$ & & & & $\begin{array}{l}(0.8 \\
2.6)\end{array}$ & & & & & & $\begin{array}{l}(0.9 \\
11.7)\end{array}$ & \\
\hline & $\geq 1$ & 53 & 41 & $\begin{array}{l}1.0 \\
\text { (ref) }\end{array}$ & - & - & - & 44 & 28 & $\begin{array}{l}1.0 \\
\text { (ref) }\end{array}$ & - & - & - & 44 & 4 & $\begin{array}{l}1.0 \\
\text { (Ref) }\end{array}$ & - \\
\hline$H R$ hazard ra & $B M$ brain & the & sic & הקחי & e $\mathrm{fr}$ & prim & diagr & is t & bra & metas & & & & & & & \\
\hline Bold text ind & ates statistic & al sig & ifican & & & & & & & & & & & & & & \\
\hline$f f v$ & selected $b$ & 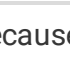 & & & 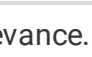 & & & & & & & & & & & & \\
\hline
\end{tabular}

\section{Intracranial distant progression and intracranial local failure}

IDP and ILF were analysed in 81 patients (81\%) who underwent follow-up imaging, while the remaining patients died or their condition deteriorated prior to the first post-treatment MRI. Median follow-up time was 18.3 (IQR 6.2-54.8) months after GKRS for IDP and 9.8 (IQR 3.527.1) months for ILF. Forty-eight patients (59\%) had IDP, at a median of 9.4 (IQR 4.8-25.9) months from GKRS. Age and number of BM were associated with IDP on univariable analysis. On multivariable analysis, the only factor significantly associated with IDP was a number of BM $>4$ (HR 2.2; $p=0.04)$. Eleven patients $(14 \%)$ had ILF, at a median of 75.5 (IQR 44.5-75.5) months from GKRS. None of the investigated factors were significantly associated with ILF (Table 2$)$.

\section{Systemic therapy}

In our evaluation of systemic therapy $(n=99)$, VEGF targeted TKIs were the most applied systemic therapy (67 patients; $68 \%)$. Seventeen patients $(17 \%)$ were treated with ICls and other systemic therapy categories were used in 20 patients $(20 \%)$. The 59 patients (60\%) receiving VEGF targeted TKI after GKRS had significantly longer survival as compared to patients not treated with VEGF targeted TKI after GKRS (HR 0.6; 95\%-CI 0.3-1.0; $\mathrm{p}$ $=0.04)$. In the 17 patients $(17 \%)$ who received ICI after GKRS, risk of death was lower compared with those who did not receive this treatment after GKRS, but not significantly (HR 0.5; 95\%-Cl 0.2-1.0; $p=0.05$ ). Risk of IDP was not significantly different between patients treated with the different systemic therapies (Table 3). 
Table 3

Multivariable analysis of risk of death and risk of intracranial distant progression by type of systemic therapy

\begin{tabular}{|c|c|c|c|c|c|c|c|c|c|c|c|}
\hline $\begin{array}{l}\text { Systemic } \\
\text { therapy ( } \\
=99)\end{array}$ & $\begin{array}{l}\text { Drug used } \\
\text { before and } \\
\text { after GKRS }\end{array}$ & $\begin{array}{l}\text { Drug } \\
\text { used } \\
\text { only } \\
\text { before } \\
\text { BM }\end{array}$ & $\begin{array}{l}\text { Drug } \\
\text { used } \\
\text { only } \\
\text { after } \\
\text { BM }\end{array}$ & $\begin{array}{l}\text { HR for OS } \\
\text { for drug } \\
\text { used before } \\
\text { GKRS } \\
(95 \% \mathrm{Cl})\end{array}$ & $\mathbf{P}$ & $\begin{array}{l}\text { HR for OS for } \\
\text { drug used } \\
\text { after GKRS } \\
(95 \% \mathrm{Cl})\end{array}$ & $\mathbf{P}$ & $\begin{array}{l}\text { HR for IDP } \\
\text { for drug } \\
\text { used before } \\
\text { GKRS ( } 95 \% \\
\text { Cl) }\end{array}$ & $\mathbf{P}$ & $\begin{array}{l}\text { HR for } \\
\text { IDP for } \\
\text { drug } \\
\text { used } \\
\text { after } \\
\text { GKRS } \\
(95 \% \mathrm{Cl})\end{array}$ & $\mathbf{P}$ \\
\hline $\begin{array}{l}\text { VEGF } \\
\text { targeted } \\
\text { TKI }\end{array}$ & 39 (39.4\%) & $8(8.1 \%)$ & $\begin{array}{l}20 \\
(20.2 \%)\end{array}$ & $\begin{array}{l}0.6 \\
(0.4,1.0)\end{array}$ & 0.06 & $\begin{array}{l}0.6 \\
(0.3,1.0)\end{array}$ & 0.04 & $\begin{array}{l}0.6 \\
(0.3,1.2)\end{array}$ & 0.13 & $\begin{array}{l}0.8 \\
(0.4,1.5)\end{array}$ & 0.42 \\
\hline $\begin{array}{l}\text { Immune } \\
\text { checkpoint } \\
\text { inhibitor }\end{array}$ & $3(3.0 \%)$ & $0(0.0 \%)$ & $\begin{array}{l}14 \\
(14.1 \%)\end{array}$ & $\begin{array}{l}1.3 \\
(0.3,5.3)\end{array}$ & 0.71 & $\begin{array}{l}0.5 \\
(0.2,1.0)\end{array}$ & 0.05 & $\begin{array}{l}0.4 \\
(0.1,2.9)\end{array}$ & 0.36 & $\begin{array}{l}0.7 \\
(0.3,1.4)\end{array}$ & 0.27 \\
\hline Other & $\begin{array}{l}3 \\
(3.0 \%)\end{array}$ & $7(7.1 \%)$ & $\begin{array}{l}10 \\
(10.1 \%)\end{array}$ & $\begin{array}{l}1.4 \\
(0.8,2.8)\end{array}$ & 0.27 & $\begin{array}{l}1.2 \\
(0.7,2.1)\end{array}$ & 0.60 & $\begin{array}{l}2.1 \\
(0.9,5.0)\end{array}$ & 0.11 & $\begin{array}{l}1.6 \\
(0.8,3.3)\end{array}$ & 0.21 \\
\hline
\end{tabular}

GKRS Gamma Knife radiosurgery, BM brain metastasis, HR hazard ratio, OS overall survival, IDP intracranial disease progression, VEGF vascular endothelial growth factor, $T K /$ tyrosine kinase inhibitor

Bold text indicates statistical significance.

Patients who received a type of systemic therapy before (i.e., 'before' and 'before and after') or after (i.e. 'after' and 'before and after') GKRS were compared with those who did not receive this therapy.

\section{Cause of death}

The cause of death was known in 72 of 85 deceased patients (85\%). Among those, 14 patients (19\%) died from intracranial diseases, while 39 patients (54\%) expired due to extracranial tumor activity. In 14 patients (19\%), cause of death was attributed to both, and five patients (7\%) died from intercurrent disease or euthanasia. Patients with Renal GPA scores of 0.0-1.0 mostly died from extracranial tumor activity (78\%), while this percentage was lower in the GPA categories 1.5-2.0 (22\%), 2.5-3.0 (58\%) and 3.5-4.0 (55\%) (Table 4).

Table 4

Cause of death by Renal GPA category

\begin{tabular}{|llllll|}
\hline $\begin{array}{l}\text { Cause of death } \\
(\mathbf{n}=\mathbf{8 5})\end{array}$ & Overall & GPA 0.0-1.0 & GPA 1.5-2.0 & GPA 2.5-3.0 & GPA 3.5-4.0 \\
\hline Intracranial & $14(19 \%)$ & $0(0 \%)$ & $3(33 \%)$ & $2(8 \%)$ & $6(30 \%)$ \\
\hline Extracranial & $39(54 \%)$ & $7(78 \%)$ & $2(22 \%)$ & $15(58 \%)$ & $11(55 \%)$ \\
\hline Intra- and extracranial & $14(19 \%)$ & $1(11 \%)$ & $4(44 \%)$ & $6(23 \%)$ & $2(10 \%)$ \\
\hline Intercurrent & $3(4 \%)$ & $1(11 \%)$ & $0(0 \%)$ & $1(4 \%)$ & $1(5 \%)$ \\
\hline Euthanasia & $2(3 \%)$ & $0(0 \%)$ & $0(0 \%)$ & $2(8 \%)$ & $0(0 \%)$ \\
\hline Not available & 13 & 1 & 2 & 3 & 7 \\
\hline GPA graded prognostic assessment & & & \\
\hline Percentages based on the valid, non-missing cases.
\end{tabular}

\section{Discussion}

The aim of this study is to evaluate the updated Renal GPA for patients with RCC BM treated with GKRS in an independent large real world cohort. To our knowledge, this tool was validated in one prior cohort of 23 patients treated with SRS other than GKRS [21]. Overall, our study confirms the updated Renal GPA as a valuable prognostic instrument to predict survival, as the instrument was able to divide our cohort into groups with different survival. Patients who had Renal GPA scores of 3.5-4.0 had a median survival of twenty months. This contrasts to the median survival of seven months in patients with GPA scores of < 3.5. Moreover, median survival was only three months in patients with GPA scores of $0.0-1.0$. However, $78 \%$ of patients in this lowest GPA category died from extracranial disease activity, so that we cannot conclude that GKRS is ineffective 
in this group. This finding underlines the importance of combining SRS with systemic therapy to control extracranial disease, especially as novel effective treatments have become available.

Besides validating the updated Renal GPA, we performed a multivariable analysis on the association between pre-treatment factors and OS. Factors predictive for longer OS in the updated Renal GPA cohort were higher performance status, absent extracranial disease, fewer BM and higher serum hemoglobin levels [20]. Our analysis confirms higher performance status and higher serum hemoglobin levels as factors favorable on OS. Karnofsky performance status has extensively been described as a powerful predictor of OS in RCC BM patients treated with SRS [16, 2224]. However, this is in contrast with a recent study that did not find a significant OS difference between performance status of $70-80$ vs $\geq 90$ [25]. The predictive value of serum hemoglobin for OS has exclusively been identified in the updated Renal GPA study for RCC BM patients [20]. Contrary to the updated Renal GPA cohort, lower age was an additional predictor for extended OS in our cohort. The predictive value of age has also been reported in prior cohorts $[24,26]$. In addition, extended OS was predicted by longer time from primary diagnosis to brain metastasis, which has been reported before $[24,26,27]$.

Our findings suggest that patients treated with VEGF targeted TKI after GKRS have improved OS compared to patients who did not receive this treatment. These findings are in line with a recent meta-analysis, which indicated prolonged survival for RCC BM patients treated with SRS when combined with VEGF targeted TKI [28]. However, Sperduto's cohort showed a higher death risk in patients treated with VEGF targeted TKI before radiosurgery, whereas treatment after GKRS was not related with OS difference [20].

In our cohort, ICl treatment after GKRS was associated with longer OS, although not statistically significant $(p=0.05)$. Previous studies described favorable survival outcomes in RCC BM patients treated with the combination of SRS and ICI [29]. In contrast, ICI treatment in the cohort of Sperduto et al. was not associated with longer survival [20]. A favorable effect of ICls on OS might be attributed to their extracranial effects, but there are also clues for intracranial efficacy of ICls [30]. However, the first prospective study assessing ICI activity in patients with RCC BM showed intracranial response of only 12\% [31]. In contrast, ICls are known to be efficacious on BM from melanoma [32-34]. In the era of our cohort, only $\mathrm{ICI}$ monotherapy beyond first-line was available. Larger prospective studies are needed to assess the real intracranial efficacy of ICls in BM from $\mathrm{RCC}$, with special attention to dual ICI and ICI/TKI combination therapy.

The retrospective design of our study makes it subject to selection bias and confounding. Systemic therapy selection is influenced by many factors, including performance status, comorbidity, previous systemic therapy and IMDC criteria [8], which makes it difficult to draw conclusions about causality between treatments and outcomes. Additionally, some data were missing as they had to be extracted from (external) patient files. Sufficient data were available to calculate updated Renal GPA scores in 91 patients (91\%). Patients with missing hemoglobin levels could still be assigned to a GPA category, and this may have led to misclassification of those patients. Furthermore, sample sizes were limited and especially those of patients treated with ICls, as this therapy was not yet available in the first years of our cohort. Finally, our data represent patients treated in a third-line referral center, as our center was the only one with Gamma Knife technology in The Netherlands until 2018. Therefore, they might not reflect patients in regular hospitals.

\section{Conclusion}

This study confirms the updated Renal GPA as a valuable instrument to estimate survival in patients with BM from RCC treated with GKRS. Age and time from primary diagnosis to BM were found as additional predictors of survival, while the predictive value of number of BM and extracranial disease was not detected. Both VEGF targeted TKI and immune checkpoint inhibitor treatment were associated with improved survival. Larger and preferably prospective studies are warranted to update the Renal GPA in the era of immunotherapy and to elucidate the benefits of systemic treatments on survival and intracranial tumor control.

\section{Declarations}

\section{Data availability}

The datasets used and/or analyzed during the current study are available from the corresponding author on reasonable request.

\section{Funding}

The authors received no specific funding for this work.

\section{Author information}

\section{Author affiliations:}

Department of Internal Medicine/Medical Oncology, Elisabeth-TweeSteden Hospital, Tilburg, The Netherlands 
Niels J. van Ruitenbeek, Laurens V. Beerepoot

Netherlands Comprehensive Cancer Organisation (IKNL), Utrecht, The Netherlands

Vincent K.Y. HoDepartment of Internal Medicine/Oncology, Amphia Hospital, Breda, The Netherlands

Hans M. WestgeestGamma Knife Center Tilburg, Elisabeth-TweeSteden Hospital, Tilburg, The Netherlands

Patrick E.J. Hanssens

\section{Contributions:}

Conception and design: NR, VH, LB, PH. Data collection and assembly: NR. Data analysis and interpretation: all authors. Writing the original draft: NR. Reviewing and editing: all authors. Supervision: LB, PH.

\section{Corresponding author:}

Correspondence to N.J. van Ruitenbeek, n.vanruitenbeek@etz.nl

\section{Ethics declarations}

\section{Conflict of interest:}

The authors have no conflicts of interest to declare that are relevant to the content of this article.

\section{Ethical approval:}

The authors confirm that they have read the Journal's position on issues involved in ethical publication and affirm that this report is consistent with those guidelines.

\section{Informed consent:}

Formal consent is not required for this type of research.

\section{References}

[1] Gao J, Zhao S, Halstensen TS. Increased interleukin-6 expression is associated with poor prognosis and acquired cisplatin resistance in head and neck squamous cell carcinoma. Oncol Rep. 2016;35(6):3265-3274.

[2] Bowman IA, Bent A, Le T, et al. Improved Survival Outcomes for Kidney Cancer Patients With Brain Metastases. Clin Genitourin Cancer. 2019;17(2):e263-e272.

[3] Sun M, De Velasco G, Brastianos PK, et al. The Development of Brain Metastases in Patients with Renal Cell Carcinoma: Epidemiologic Trends, Survival, and Clinical Risk Factors Using a Population-based Cohort. Eur Urol Focus. 2019;5(3):474-481.

[4] Bianchi M, Sun M, Jeldres C, et al. Distribution of metastatic sites in renal cell carcinoma: a population-based analysis. Ann Oncol. 2012;23(4):973-980.

[5] Motzer RJ, Hutson TE, Tomczak P, et al. Sunitinib versus interferon alfa in metastatic renal-cell carcinoma. N Engl J Med. 2007;356(2):115-124.

[6] Motzer RJ, Tannir NM, McDermott DF, et al. Nivolumab plus Ipilimumab versus Sunitinib in Advanced Renal-Cell Carcinoma. N Engl J Med. 2018;378(14):1277-1290.

[7] Rini BI, Plimack ER, Stus V, et al. Pembrolizumab plus Axitinib versus Sunitinib for Advanced Renal-Cell Carcinoma. N Engl J Med. 2019;380(12):1116-1127.

[8] Heng DY, Xie W, Regan MM, et al. Prognostic factors for overall survival in patients with metastatic renal cell carcinoma treated with vascular endothelial growth factor-targeted agents: results from a large, multicenter study. J Clin Oncol. 2009;27(34):5794-5799.

[9] Bennani O, Derrey S, Langlois O, et al. Brain metastasis from renal cell carcinoma. Neurochirurgie. 2014;60(1-2):12-16.

[10] Vickers MM, Al-Harbi H, Choueiri TK, et al. Prognostic factors of survival for patients with metastatic renal cell carcinoma with brain metastases treated with targeted therapy: results from the international metastatic renal cell carcinoma database consortium. Clin Genitourin 
Cancer. 2013;11(3):311-315.

[11] Blanco Al, Teh BS, Amato RJ. Role of radiation therapy in the management of renal cell cancer. Cancers (Basel). 2011;3(4):4010-4023.

[12] Patil CG, Pricola K, Sarmiento JM, Garg SK, Bryant A, Black KL. Whole brain radiation therapy (WBRT) alone versus WBRT and radiosurgery for the treatment of brain metastases. Cochrane Database Syst Rev. 2017;9:Cd006121.

[13] Chang EL, Wefel JS, Hess KR, et al. Neurocognition in patients with brain metastases treated with radiosurgery or radiosurgery plus whole-brain irradiation: a randomised controlled trial. Lancet Oncol. 2009;10(11):1037-1044.

[14] Aoyama $\mathrm{H}$, Shirato $\mathrm{H}$, Tago $\mathrm{M}$, et al. Stereotactic radiosurgery plus whole-brain radiation therapy vs stereotactic radiosurgery alone for treatment of brain metastases: a randomized controlled trial. JAMA. 2006;295(21):2483-2491.

[15] Wardak Z, Christie A, Bowman A, et al. Stereotactic Radiosurgery for Multiple Brain Metastases From Renal-Cell Carcinoma. Clin Genitourin Cancer. 2019;17(2):e273-e280.

[16] Kano H, lyer A, Kondziolka D, Niranjan A, Flickinger JC, Lunsford LD. Outcome predictors of gamma knife radiosurgery for renal cell carcinoma metastases. Neurosurgery. 2011;69(6):1232-1239.

[17] Kondziolka D, Parry PV, Lunsford LD, et al. The accuracy of predicting survival in individual patients with cancer. J Neurosurg. 2014;120(1):24-30.

[18] Sperduto PW, Kased N, Roberge D, et al. Summary report on the graded prognostic assessment: an accurate and facile diagnosis-specific tool to estimate survival for patients with brain metastases. J Clin Oncol. 2012;30(4):419-425.

[19] Yamamoto M, Serizawa T, Sato Y, et al. Validity of two recently-proposed prognostic grading indices for lung, gastro-intestinal, breast and renal cell cancer patients with radiosurgically-treated brain metastases. J Neurooncol. 2013;111(3):327-335.

[20] Sperduto PW, Deegan BJ, Li J, et al. Estimating survival for renal cell carcinoma patients with brain metastases: an update of the Renal Graded Prognostic Assessment tool. Neuro Oncol. 2018;20(12):1652-1660.

[21] Nagtegaal SHJ, Claes A, Suijkerbuijk KPM, Schramel F, Snijders TJ, Verhoeff JJC. Comparing survival predicted by the diagnosis-specific Graded Prognostic Assessment (DS-GPA) to actual survival in patients with 1-10 brain metastases treated with stereotactic radiosurgery. Radiother Oncol. 2019;138:173-179.

[22] Guseľnova K, Lishchak R, Shimonova G, et al. Results of gamma-knife radiosurgery treatment for intracranial metastases of renal-cell cancer and prognostic factors influencing on the survival (joint study of three Eastern European Centers). Zh Vopr Neirokhir Im N N Burdenko. 2013;77(6):4-12; discussion 13.

[23] Shuto T, Inomori S, Fujino H, Nagano H. Gamma knife surgery for metastatic brain tumors from renal cell carcinoma. J Neurosurg. 2006;105(4):555-560.

[24] Sheehan JP, Sun MH, Kondziolka D, Flickinger J, Lunsford LD. Radiosurgery in patients with renal cell carcinoma metastasis to the brain: long-term outcomes and prognostic factors influencing survival and local tumor control. J Neurosurg. 2003;98(2):342-349.

[25] Juloori A, Miller JA, Parsai S, et al. Overall survival and response to radiation and targeted therapies among patients with renal cell carcinoma brain metastases. J Neurosurg. 2019:1-9.

[26] Klausner G, Troussier I, Biau J, et al. Stereotactic Radiation Therapy for Renal Cell Carcinoma Brain Metastases in the Tyrosine Kinase Inhibitors Era: Outcomes of 120 Patients. Clin Genitourin Cancer. 2019;17(3):191-200.

[27] Yildiz I, Bilici A, Karadurmuş N, et al. Prognostic factors for survival in metastatic renal cell carcinoma patients with brain metastases receiving targeted therapy. Tumori. 2018;104(6):444-450.

[28] Khan M, Zhao Z, Arooj S, Liao G. Impact of Tyrosine Kinase Inhibitors (TKIs) Combined With Radiation Therapy for the Management of Brain Metastases From Renal Cell Carcinoma. Front Oncol. 2020;10:1246.

[29] Chen L, Douglass J, Kleinberg L, et al. Concurrent Immune Checkpoint Inhibitors and Stereotactic Radiosurgery for Brain Metastases in Non-Small Cell Lung Cancer, Melanoma, and Renal Cell Carcinoma. Int J Radiat Oncol Biol Phys. 2018;100(4):916-925.

[30] Kattan J, Rassy EE, Assi T, Bakouny Z, Pavlidis N. A comprehensive review of the role of immune checkpoint inhibitors in brain metastasis of renal cell carcinoma origin. Crit Rev Oncol Hematol. 2018;130:60-69.

Page 13/14 
[31] Flippot R, Dalban C, Laguerre B, et al. Safety and Efficacy of Nivolumab in Brain Metastases From Renal Cell Carcinoma: Results of the GETUG-AFU 26 NIVOREN Multicenter Phase II Study. J Clin Oncol. 2019;37(23):2008-2016.

[32] Long GV, Atkinson V, Lo S, et al. Combination nivolumab and ipilimumab or nivolumab alone in melanoma brain metastases: a multicentre randomised phase 2 study. Lancet Oncol. 2018;19(5):672-681.

[33] Tawbi HA, Forsyth PA, Algazi A, et al. Combined Nivolumab and Ipilimumab in Melanoma Metastatic to the Brain. N Engl J Med. 2018;379(8):722-730.

[34] Rulli E, Legramandi L, Salvati L, Mandala M. The impact of targeted therapies and immunotherapy in melanoma brain metastases: A systematic review and meta-analysis. Cancer. 2019;125(21):3776-3789.

Figures

A Overall survival by GPA category

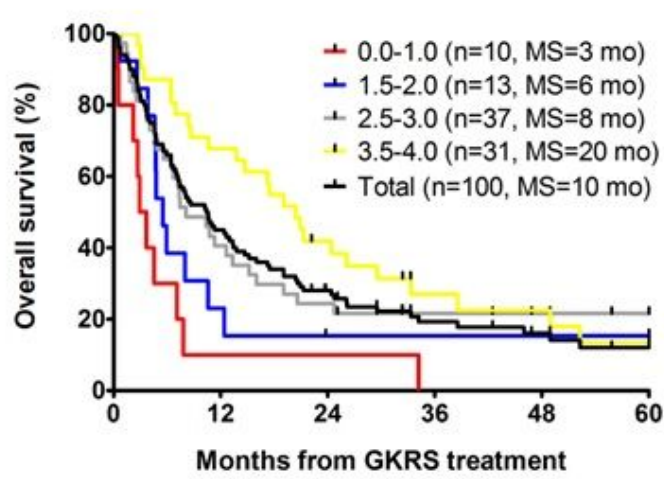

C Overall survival GPA $0.0-3.0$ vs $3.5-4.0$

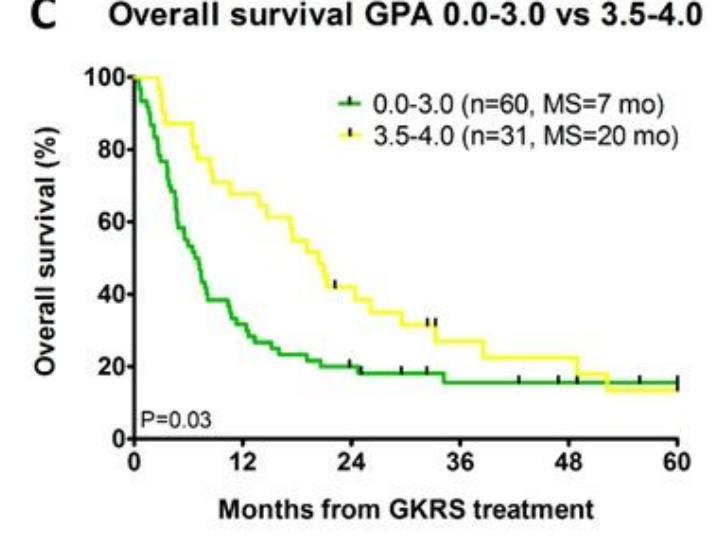

Figure 1

Kaplan-Meier curves showing overall survival by (a) Renal GPA category (b) Renal GPA 0.0-1.0 vs > 1.0 (c) Renal 3.5-4.0 vs < 3.5.

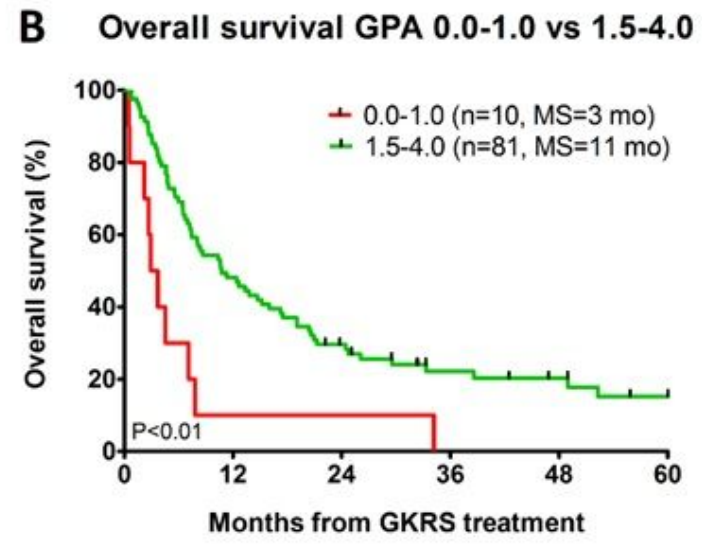

Months from GKRS treatment 